\title{
Development of a palm olein oil-in-water (o/w) emulsion stabilized by a whey protein isolate nanofibrils-alginate complex
}

\begin{abstract}
An oil-in-water $(\mathrm{o} / \mathrm{w})$ emulsion is a system where the oil droplets are dispersed within a watery phase. The most important function of emulsions is their ability to incorporate lipophilic components into food matrices. Thus, it is crucial to develop an emulsion that is highly stable. This work was aimed at developing a palm olein o/w emulsion stabilized by a whey protein isolate nanofibrils-alginate complex, as well as evaluating the influence of oil load and the homogenization process (both pressure and cycle) on the characteristics of the o/w emulsions. Emulsions were analyzed for droplet size, zeta potential, viscosity, creaming stability, and morphology. The results showed that an increase in oil load led to a larger droplet size, less negative zeta potential, and emulsions that were more viscous and less stable. On the other hand, increasing homogenization pressure and the number of homogenization cycles resulted in a smaller droplet size, more negative zeta potential, and emulsions that were less viscous and more stable. Emulsions with a smaller droplet size and better stability resulted from lower oil load, high homogenization pressure and more homogenization cycles.
\end{abstract}

Keyword: Whey protein isolate; Nanofibrils; Alginate; Complexation; Homogenization 